\title{
Mechanism and disease implications of necroptosis and neuronal inflammation
}

\author{
Sara R. Oliveira', Joana D. Amaral (1) and Cecília M. P. Rodrigues ${ }^{1}$
}

Necroptosis is a form of regulated necrotic cell death, executed via activation of receptor-interacting protein 1 (RIP1) and 3 (RIP3), which is activated under apoptosisdeficient conditions. Although necroptosis could be initiated by several stimuli, the activation mediated by death receptors, particularly tumor necrosis factor receptor 1 (TNFR1), is the most widely studied ${ }^{1}$. At the molecular level, this type of cell death includes auto- and trans-phosphorylation of RIP1 and RIP3, which leads to the assembly of an amyloid-like multiprotein complex, socalled necrosome ${ }^{1}$. In addition to RIP1 and RIP3, mixed lineage kinase domain-like (MLKL) pseudokinase is also involved in necroptosis, being recruited and phosphorylated at T357/S358 by RIP3. Upon phosphorylation, MLKL oligomerizes and migrates from the cytoplasm to the cell membrane, thus causing necrotic membrane disruption and cell death ${ }^{2}$.

Growing evidence shows that necroptosis is a key event in the pathogenesis of several diseases with an inflammatory component. Regulated necrosis was first investigated in ischemic brain injury in $2005^{3}$, and later in liver injury ${ }^{4}$ but also in neurodegenerative diseases. In fact, necroptosis has been implicated in Huntington's disease ${ }^{5}$, multiple sclerosis ${ }^{6}$, Alzheimer's disease ${ }^{7}$ and, more recently, in Parkinson's disease ${ }^{8}$. Of importance is the fact that genetic or chemical blockage of necroptosis results in disease amelioration. Pharmacological inhibition of necroptosis was first investigated using necrostatin-1 (Nec-1), an allosteric inhibitor of RIP1, which stabilizes a specific inactive conformation of the kinase domain ${ }^{9}$. However, in vivo studies with this molecule were limited due to its poor pharmacokinetic properties, including a short half-life of approximately $1 \mathrm{~h}$, along with reduced solubility. In addition, Nec-1 presented off-target activity,

\footnotetext{
Correspondence: Cecília M. P. Rodrigues (cmprodrigues@ff.ulisboa.pt) ${ }^{1}$ Research Institute for Medicines (iMed.ULisboa), Faculty of Pharmacy, Universidade de Lisboa, Lisbon 1649-003, Portugal
}

inhibiting indoleamine 2,3-dioxygenease (IDO), an enzyme involved in adaptive and innate immune responses ${ }^{9}$. To overcome these limitations, Nec-1 molecule was further optimized, leading to the development of necrostatin-1 stable (Nec-1s), selective for RIP1-kinase inhibition, but still with poor pharmacokinetic properties ${ }^{9}$. Other molecules targeting different components of the necroptotic signaling pathway were also described, including GSK'872 and necrosulfonamide that inhibit RIP3 and MLKL, respectively. Nevertheless, all of them presented several limitations. GSK2982772, a RIP1-kinase inhibitor, is currently in phase 2a clinical studies for psoriasis, rheumatoid arthritis, and ulcerative colitis ${ }^{10}$, which highlights the relevance and importance of pharmacological inhibition of necroptosis in the context of disease.

In our recent paper published in Cell Death Discovery ${ }^{11}$, we screened a small in-house library of molecules for their ability to inhibit necroptosis after successful method development using an in vitro model of microglia necroptosis, based on the murine BV2 microglia cell line (Fig. 1). The phenotypic screening identified a new oxazolone-Oxa12-that strongly inhibits necroptosis in two different cellular models-BV2 and L929 cells-without cytotoxicity associated. Further, Oxa12 inhibited important markers of necroptosis commitment, including necrosome assembly and MLKL S358 phosphorylation in BV2 cells. Of note, in silico molecular docking calculations for Oxa12 inside the RIP1 kinase domain revealed, that without any constraint, Oxa12 is occupying a region similar to the co-crystallized inhibitor. Oxa12, however, is slightly rotated in the binding pocket when compared with the crystallographic ligand, being close to Asp156, Leu157, Met67, and Met95, which may enable important hydrogen bonds and $\pi$ interactions. Oxa12 showed slightly increased interaction distances compared with the crystallographic inhibitor. 


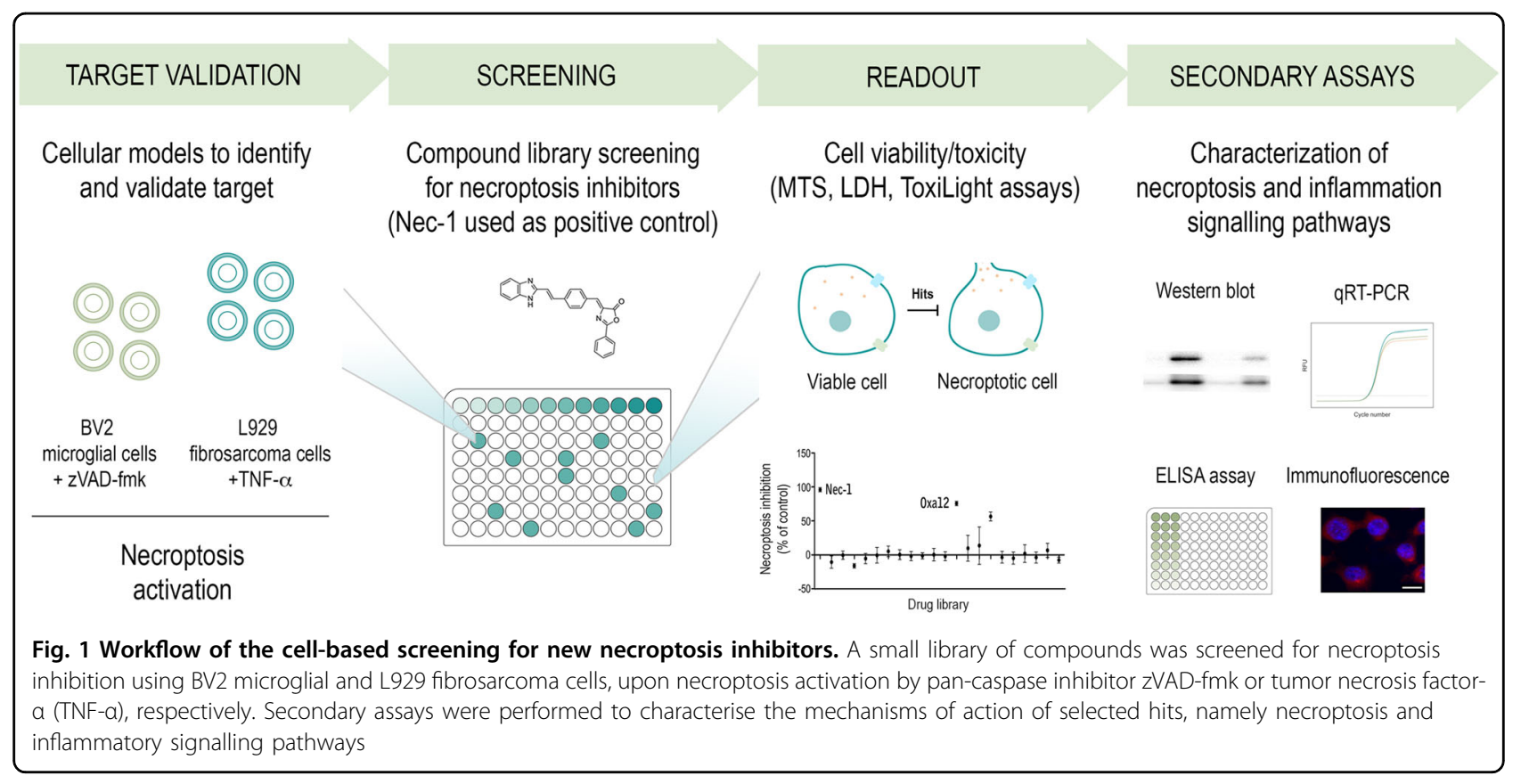

The crosstalk between necroptosis and inflammation has been a matter of debate in the past years. In fact, necroptosis was first described as a proinflammatory form of cell death culminating in the release of intracellular components, called damage-associated molecular patterns (DAMPs), to the extracellular space. Other studies suggest that necroptosis mediated by TNF- $\alpha$ may promote inflammation by a cell-autonomous mechanism involving activation of the transcription factor NF- $\mathrm{kB}$ and p38 MAPK signaling pathway, instead of direct DAMP release $^{12}$. In our work, we showed that Oxa12 is capable of reducing TNF- $\alpha$ and IL-1 $\beta$ expression levels, after cell stimulation with both necroptotic and inflammatory stimuli. We further investigated which inflammatory pathways were modulated by Oxa12 and concluded that this molecule strongly reduces necroptosis-mediated activation of two important MAPK signaling pathways, JNK and $\mathrm{p} 38$, as well as NF- $\mathrm{kB}$ activation. Our results are in accordance with previous studies, where JNK activation appears as an important player during zVAD-fmkinduced necroptosis in L929 cells, promoting TNF- $\alpha$ gene expression ${ }^{13}$. Importantly, JNK and p38 MAPK signalling pathways are involved in the pathogenesis of Alzheimer's and Parkinson's disease, where they were shown to play a role in inflammation and neurodegeneration $^{14}$. In this regard, NF- $\mathrm{kB}$ activation in glial cells appears to mediate pathological inflammatory processes, while its activation in neurons protects against neurodegeneration. Therefore, inhibition of necroptosis specifically in microglia cells may be beneficial by reducing neuroinflammation and improving neural survival in the context of disease. As an example, reduced activation of caspase-8 with consequent induction of necroptosis and inflammation has been reported in microglia cells of patients with multiple sclerosis. Importantly, this phenotype appears to contribute to disease progression ${ }^{6}$. Further, necroptosis in retina microglia promotes and amplifies inflammation, which contributes to neuronal degeneration ${ }^{15}$. In both cases, necroptosis blockade appears to reduce inflammation, rescue degeneration, and prevent neural injury both in vitro and in vivo.

In summary, our study identifies a strong lead necroptosis inhibitor-Oxa12-that is efficient at reducing necroptosis-driven inflammation as well as inflammation per se. We consider this new oxazolone a promising candidate molecule for targeting pathologies involving abnormal cell death with an inflammatory component, such as neurodegenerative diseases. In support of this idea, Oxa12 will undergo further medicinal chemistry optimization to then be tested in vivo using relevant models of disease.

\section{Acknowledgements \\ This work was supported by Programme grant SAICTPAC/0019/2015 funded by European Structural and Investment Funds through the COMPETE Programme and by National Funds through Fundação para a Ciência e a Tecnologia (FCT); iMed.ULisboa funded by FCT (UID/DTP/04138/2013); and fellowships funded by FCT (SFRH/BPD/100961/2014, PD/BD/128332/2017, SFRH/BD/102771/2014, and SFRH/BD/110672/2015).}

\section{Conflict of interest}

The authors declare that they have no conflict of interest.

\section{Publisher's note}

Springer Nature remains neutral with regard to jurisdictional claims in published maps and institutional affiliations. 
Received: 27 June 2018 Revised: 8 July 2018 Accepted: 12 July 2018 Published online: 05 September 2018

\section{References}

1. Li, J. et al. The RIP1/RIP3 necrosome forms a functional amyloid signaling complex required for programmed necrosis. Cell 150, 339-350 (2012).

2. Wang, H. et al. Mixed lineage kinase domain-like protein MLKL causes necrotic membrane disruption upon phosphorylation by RIP3. Mol. Cell 54, 133-146 (2014).

3. Degterev, A. et al. Chemical inhibitor of nonapoptotic cell death with therapeutic potential for ischemic brain injury. Nat. Chem. Biol. 1, 112-119 (2005).

4. Afonso, M. B. et al. Necroptosis is a key pathogenic event in human and experimental murine models of non-alcoholic steatohepatitis. Clin. Sci. (Lond.) 129, 721-739 (2015).

5. Vandenabeele, P., Galluzzi, L., Vanden Berghe, T. \& Kroemer, G. Molecular mechanisms of necroptosis: an ordered cellular explosion. Nat. Rev. Mol. Cell Biol. 11, 700-714 (2010)

6. Ofengeim, D. et al. Activation of necroptosis in multiple sclerosis. Cell Rep. $\mathbf{1 0}$ 1836-1849 (2015)

7. Caccamo, A. et al. Necroptosis activation in Alzheimer's disease. Nat. Neurosci. 20, 1236-1246 (2017)
8. Iannielli, A. et al. Pharmacological inhibition of necroptosis protects from dopaminergic neuronal cell death in Parkinson's disease models. Cell Rep. 22, 2066-2079 (2018)

9. Takahashi, N. et al. Necrostatin-1 analogues: critical issues on the specificity, activity and in vivo use in experimental disease models. Cell Death Dis. 3, e437 (2012).

10. Harris, P. A. et al. Discovery of a first-in-class receptor interacting protein 1 (RIP1) kinase specific clinical candidate (GSK2982772) for the treatment of inflammatory diseases. J. Med. Chem. 60, 1247-1261 (2017).

11. Oliveira S. R. et al. Phenotypic screening identifies a new oxazolone inhibitor of necroptosis and neuroinflammation. Cell Death Discov. 5, 10 (2018).

12. Zhu, K. et al. Necroptosis promotes cell-autonomous activation of proinflammatory cytokine gene expression. Cell Death Dis. 9, 500 (2018).

13. $W u, Y . T$. et al. ZVAD-induced necroptosis in L929 cells depends on autocrine production of TNFalpha mediated by the PKC-MAPKs-AP-1 pathway. Cell Death Differ. 18, 26-37 (2011).

14. Hashimoto, M. et al. Role of protein aggregation in mitochondrial dysfunction and neurodegeneration in Alzheimer's and Parkinson's diseases. Neuromolec. Med. 4, 21-36 (2003).

15. Huang, Z. et al. Necroptosis in microglia contributes to neuroinflammation and retinal degeneration through TLR4 activation. Cell Death Differ. 25 180-189 (2018) 